\section{Obstetricia \\ RESSUSCITAÇÃO INTRA-UTERINA NO TRABALHO DE PARTO: EXISTE FACTIBILIDADE ATUALMENTE NO BRASIL?}

A banalização da cesárea, em sua acepção ampla, desloca para planos ínfimos de importância as manobras e as técnicas' existentes para reverter quadros de possível depressão fetal, durante o trabalho de parto, recursos esses elaborados para se concretizar um parto por via vaginal. $O$ aprimoramento da tecnologia de vigilância do bem-estar fetal ${ }^{2}$, aplicada durante esse curto período da gestação, facilitou sobremaneira a instituição de cuidados preventivos e terapêuticos porque as ferramentas disponibilizadas permitem a deteç̧ão precoce dos desvios agudos na fisiologia feto e uteroplacentária. Entretanto, observa-se que, ao avesso de se aproveitarem dessas vantagens para o estabelecimento de medidas corretivas, ao reconhecerem quaisquer irregularidades, os obstetras brasileiros utilizam-nas como subterfúgio para a indicação imediata da via abdominal de nascimento, a despeito das eloqüentes taxas de falsos-positivos (até 80\%) exibidas pelos testes aplicados ${ }^{2}$. Embora essas anormalidades do trabalho de parto sejam responsáveis por número irrisório de casos com desfecho tão crítico e sua ocorrência seja pouco freqüente, a paralisia cerebral ${ }^{3}$ e outras seqüelas neurológicas pós-natais são preocupações constantes nas atividades em Centro Obstétrico. De fato, as complicações profissionais resultantes disso, na esfera civil e judicial implicam grande ônus emocional e, às vezes, econômico. Em face disso, é oportuno, mesmo que soe anacrônico e de pouca factibilidade, enfatizar as medidas fundadas para a correção da hipoxia intraparto. Por óbvio, é desnecessário salientar que uma instrumentalização mínima é obrigatória. Nesse sentido, a cardiotocografia (CTG) é soberana para a monitorização da freqüência cardíaca fetal (FCF) e compõe a metodologia primária para essa finalidade, empregada na maioria dos centros obstétricos dos países desenvolvidos e nos centros nacionais mais bem estruturados. Nos resultados nãotranqüilizadores desse exame, idealmente pode-se complementar a análise da vitalidade fetal por meio da gasometria ${ }^{4}$, oximetria ou pela eletrocardiografia fetal ${ }^{2}$. Se, porventura, existirem fortes indícios de hipoxia fetal, mesmo na indisponibilidade de métodos complementares à CTG, deve-se instituir os procedimentos de ressuscitação intra-uterina constituídos por: a) reposicionamento materno, nas situações de inadequações hemodinâmicas maternas e mal posicionamento do cordão umbilical (desacelerações variáveis da F(F); b) redução da atividade uterina, suspendendo a infusão de ocitocina ou ministrando uterolíticos; c) infusão de fluido intravenoso, seja solução fisiológica ou de ringer-lactato, mesmo não havendo evidências de déficit volumétrico materno; d) administração de oxigênio, de preferência, por meio de máscaras especiais, tipo "non-rebreather facemask"; e) amnioinfusão para corrigir os efeitos das compressões funiculares; f) suspensão temporária dos esforços expulsivos (puxos) até a recuperação da reserva fetal em oxigênio'. Nada a obstar à inexistência de consenso a respeito da eficácia de cada medida de ressuscitação ou de todo o conjunto de manobras se o profissional tiver o escopo franco de propiciar à parturiente um parto vaginal, a correta observância de todos os passos diagnósticos e terapêuticos pertinentes é imprescindível e, seguramente, pode coroar de êxito o seu propósito. Portanto, a factibilidade da reanimação intra-uterina está diretamente vinculada não só à cognição e domínio do obstetra em relação à propedêutica do bem-estar fetal durante o período intraparto, mas também à sua verdadeira intenção na assistência à parturiente.

Seizo MiYadahira SEIZO MIYADAHIRA Marcelo Zugaib

Referências

I.Simpson KR. Intrauterine resuscitation during labor: review of current methods and supportive evidence. J Midwifery Women's Health. 2007;52:229-37.

2.Norén H, Blad S, Carlsson A, Flisberg A, Gustavsson A, Lilja H, et al. STAN in clinical practice: The outcome of 2 years of regular use in the city of Gothenburg. Am J Obstet Gynecol. 2007; 195:7- I5.

3.Strijbis EMM, Oudman I, Van Essen P. MacLennan A. Cerebral palsy and the application of International Criteria for Acute Intrapartum Hypoxia. Obstet Gynecol. 2006; 107:1357-65.

4.Ross MG, Gala R. Use of umbilical artery base excess: algorithm for the timing of hypoxic injury. Am J Obstet Gynecol. 2002; I 87: I-9

Medicina Farmacêutica

\section{QUAL A IMPORTÂNCIA DA ANÁLISE ORÇAMENTÁRIA DE PROJETOS DE PESQUISA CLÍNICA?}

A Resolução CNS 196/96, assim como a RDC 219/04, consideram o orçamento financeiro de estudos clínicos como um dos itens que compõem o projeto de pesquisa ${ }^{1-2}$. A análise criteriosa deste documento, a fim de verificar se os custos oferecidos pelo patrocinador (em geral, a indústria farmacêutica) satisfazem os custos institucionais para a realização do estudo, deve ser, pois, uma prática comum às instituições que conduzem pesquisa clínica, devendo, sempre que possível, ser realizada por equipe especializada. Saber defender os direitos da instituição no que tange aos custos de estudos clínicos demonstra bom preparo da instituição e certamente é apreciado pelos patrocinadores ${ }^{3}$, além de ser parte fundamental na estratégia de manutenção da sustentabilidade dos centros de pesquisa.

O orçamento do estudo deve contemplar os custos diretos, como honorários profissionais, testes diagnósticos e terapêuticos, procedimentos cirúrgicos, custos administrativos, devendo ainda contemplar as despesas com transporte e alimentação a serem reembolsadas pelo paciente. Adicionalmente, custos indiretos também devem ser incluídos no orçamento; estes se referem aos custos não atribuíveis diretamente ao estudo e que, por este motivo, são de difícil mensuração e usualmente esquecidos. Uma alternativa, utilizada por boa parte 
das instituições, é a inclusão de valor adicional, por vezes denominado overhead, que pode variar de $20 \%$ a $40 \%$ do valor atribuído aos custos diretos e visa a cobrir tais despesas ${ }^{3}$.

A análise orçamentária deve ser baseada no fluxograma do estudo e nos valores cobrados na instituição em que o estudo será desenvolvido. Questões como fornecimento de medicamentos do estudo, mesmo após o seu término, pagamento de triagem de pacientes que não puderam ser incluídos no estudo (screening failure), pagamento de exames adicionais ou que sejam necessários no caso de complicações devem ser previamente acordadas entre patrocinador e instituição e devem ser consideradas ao se proceder a análise. Além disso, as dificuldades mais comumente observadas incluem: falta de ferramentas que facilitem e padronizem o processo, falta de informações e objetividade na descrição de alguns procedimentos, imprecisão de custos hospitalares, interação insuficiente entre os diferentes departamentos da instituição e dificuldade na padronização do repasse da verba do estudo aos diferentes serviços envolvidos.

O processo de análise orçamentária é necessário e importante para o adequado andamento de qualquer projeto de pesquisa. Deve ser conduzido antes da assinatura do contrato entre o patrocinador e a instituição para que não restem pendências a serem resolvidas após o início da fase de inclusão de pacientes no estudo.

Mariana Bueno Denise Batista Nunes Sonia Mansoldo Dainesi

Referências

I. Brasil. Conselho Nacional de Saúde (CNS). Resolução I 96/96 [citado 30 out 2007]. Disponível em: http://www.conselho.saude.gov.br/comissao/conep/ resolucao.html.

2. Brasil. Agência Nacional de Vigilância Sanitária (ANVISA). Resolução RDC 219/04 [citado 30 out 2007]. Disponível em:

http://e-legis. anvisa.gov.br/leisref/public/showAct. php?id= $17042 \&$ word $=$.

3. Blanchard A, Maffei L. The real cost of a clinical trial. GCPJ 2005; : 25-8.

\section{Ginecalagia}

\section{TROMBOEMBOLISMO E CÂNCER DE MAMA:' QUANDO INDICAR A PROFILAXIA MEDICAMENTOSA?}

O câncer de mama, excluindo-se os cânceres de pele, é em nosso meio a neoplasia maligna que mais acomete a mulher, estimando-se 48.930 novos casos para 2006!

O tratamento do câncer de mama se fundamenta na cirurgia, quimioterapia, hormonioterapia e radioterapia, sendo que todas essas modalidades aumentam 0 risco de tromboemboembolismo.

O tromboembolismo venoso (TEV) - tanto a trombose venosa profunda (TVP) como o tromboembolismo pulmonar representa uma importante causa de morbi-mortalidade em pacientes com câncer.

De fato, a estimativa anual de um primeiro episódio de TEV na população geral é de $0,117 \% \%^{2}$, enquanto que naquela com câncer o risco é quatro vezes maior, a depender do tipo de câncer².

No tocante ao câncer de mama, o risco de TEV é 9 a 12 vezes maior do que o da população geral ${ }^{3}$, decorrente não só da doença, mas também da cirurgia, quimioterapia, hormonioterapia, radioterapia e do uso de cateteres centrais. A associação quimioterapia/hormonioterapia revelou $9 \%$ na taxa de TEV, enquanto a hormonioterapia isolada desvelou risco duas a cinco vezes maior. A cirurgia por si mostrou risco duas vezes maior de TVP e três vezes de TEP².

Estudo envolvendo mais de 100.000 mulheres com câncer de mama apontou incidência cumulativa de TEV de I,2\%, com aumento da mortalidade ${ }^{4}$. Com o intuito de reduzir o risco de TEV, a American Society of Clinical Oncology 5 propôs o seguinte guideline:

I. Em pacientes submetidas ao tratamento cirúrgico de doença primária: prescrever precocemente heparina não fracionada (HNF) ou HBPM (heparina de baixo peso molecular) ou fondaparinux e mantê-la por 7 a 10 dias;

2. Nas pacientes hospitalizadas com doença ativa, sem sangramento ou com contra-indicação ao uso de anticoagulantes, empregar a HNF ou HBPM ou fondaparinux durante a internação;

3. Nos casos de TEV estabelecido, a melhor escolha é HBPM por período não inferior a seis meses;

4. Em pacientes sob tratamento quimioterápico ambulatorial, não indicar a profilaxia de rotina;

5. Não há evidências suficientes para se prescrever anticoagulantes na ausência de TEV visando o aumento da sobrevida.

A publicação deste guideline da ASCO reforça a necessidade de se estabelecer protocolos de conduta bem estruturados, visando a prevenção de doenças que, quando negligenciadas, podem determinar sérios comprometimentos à saúde dos pacientes.

\section{Vilmar M. de Oliveira \\ TSUTOMU AoKI \\ José M. AldRighI}

Referências

I. Brasil. Ministério da Saúde. INCA/Comprev. Estimativa 2006: Incidência de câncer no Brasil. Rio de Janeiro; 2005.

2. Andtbacka RH, Babiera G, Singletary SE, Hunt KK, Meric-Bernstam F, Feig BW, et al. Incidence and prevention of venous thromboembolism in patients undergoing breast cancer surgery and treated according to clinical pathways. Ann Surg. 2006;243(1):96-101.

3. Heit JA. Cancer and venous thromboembolism: scope of the problem.

4. Cancer Control. 2005; I2(Suppl I):5-10.

Incidence of venous thromboembolism and the impact on survival in breast cancer patients. J Clin Oncol. 2007;25(I):70-6.

5. Lyman GH, Khorana AA, Falanga A, Clarke-Pearson D, Flowers C, Jahanzeb $M$, et al. American Society of Clinical Oncology Guideline: recommendations for venous thromboembolism prophylaxis and treatment in patients with cancer. J Clin Oncol. 2007;25(34): I - 16 